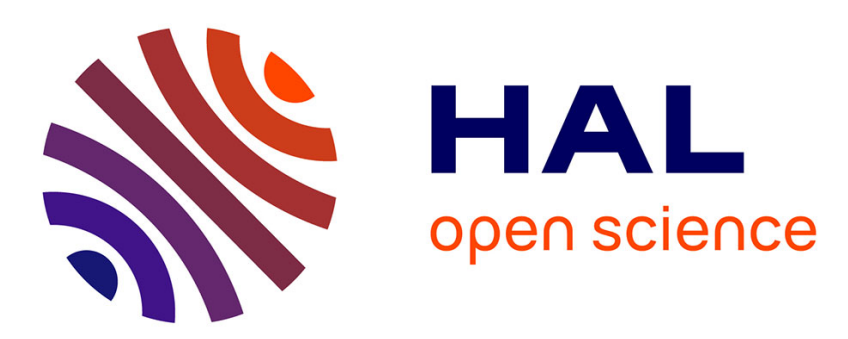

\title{
SEU sensitivity of Junctionless Single-Gate SOI MOSFETs-based 6T SRAM cells investigated by 3D TCAD simulation
}

Daniela Munteanu, Jean-Luc Autran

\section{- To cite this version:}

Daniela Munteanu, Jean-Luc Autran. SEU sensitivity of Junctionless Single-Gate SOI MOSFETsbased 6T SRAM cells investigated by 3D TCAD simulation. Microelectronics Reliability, 2015, 55, pp.1501 - 1505. 10.1016/j.microrel.2015.06.107 . hal-01429427

\section{HAL Id: hal-01429427 \\ https://hal-amu.archives-ouvertes.fr/hal-01429427}

Submitted on 8 Jan 2017

HAL is a multi-disciplinary open access archive for the deposit and dissemination of scientific research documents, whether they are published or not. The documents may come from teaching and research institutions in France or abroad, or from public or private research centers.
L'archive ouverte pluridisciplinaire HAL, est destinée au dépôt et à la diffusion de documents scientifiques de niveau recherche, publiés ou non, émanant des établissements d'enseignement et de recherche français ou étrangers, des laboratoires publics ou privés. 


\title{
SEU Sensitivity of Junctionless Single-Gate SOI MOSFETs-based 6T SRAM Cells Investigated by 3D TCAD Simulation
}

\author{
D. Munteanu ${ }^{\mathrm{a}}$, J.L. Autran ${ }^{\mathrm{a}, *}$ \\ ${ }^{a}$ Aix-Marseille University \& CNRS, IM2NP (UMR 7334), Faculté des Sciences - Service 142, \\ Avenue Escadrille Normandie Niémen, F-13397 Marseille Cedex 20, France
}

\begin{abstract}
The Junctionless (JL) Single-Gate SOI (JL-SOI) technology is potentially interesting for future ultra-scaled devices, due to a simplified technological process and reduced leakage currents. In this work, we investigate the radiation sensitivity of JL-SOI MOSFETs and 6T SRAM cells. A detailed comparison with JL Double-Gate (JL-DG), inversion-mode (IM) SOI (IM-SOI), and IM-DG MOSFETs has been performed. 3-D simulations indicate that JL-SOI MOSFETS and SRAM cells are naturally less immune to radiation than the other structures.
\end{abstract}

\section{Keywords}

Junctionless Single-Gate SOI MOSFET, SEU, SEE, bipolar amplification, threshold LET SRAM cell, Double-Gate MOSFET, SOI

\section{*Corresponding author:}

Prof. Jean-Luc Autran

IM2NP - UMR CNRS 7334

Faculté des Sciences - Service 142

Avenue Escadrille Normandie - F-13397

Marseille Cedex 20 France

Phone: + $33(0) 413594627$

Fax: +33 (0)491288531

Email: jean-luc.autran@univ-amu.fr 


\section{Introduction}

As the MOSFET is scaling down, the sensitivity of integrated circuits to radiation coming from space or present in the terrestrial environment has been found to seriously increase [1]. For ultra-scaled devices entering in the area of nanoelectronics, natural radiation at ground level is presently inducing one of the highest failure rates of all reliability concerns [2]. In particular, ultra-scaled memory integrated circuits have been found to be more sensitive to single-event-upset (SEU) induced by ionizing particles.

To meet the roadmap requirements in the nanometre scale, several promising technological solutions have been proposed, such as the Fully-depleted Single-Gate SOI technologies (FDSOI) fabricated with ultra-thin silicon bodies [3]. FDSOI devices show enhanced performances in terms of channel potential control, reducing short-channel (SCE) and floating body effects (FBE). A new concept of MOSFET without junctions, called junctionless (JL) MOSFET has been proposed these last years and experimentally validated [4-7]. A JL MOSFET designed with a single-gate SOI structure (JL-SOI, Fig. 1a) is an SOI transistor with the same type of semiconductor throughout the entire silicon film, including the source, channel and drain regions. JL-SOI devices present a real advantage since their fabrication process is simplified compared to the conventional process: there are no doping gradients in the device [6] and no semiconductor-type inversion. In addition, the junction leakage currents are totally suppressed and the off-state current $\left(\mathrm{I}_{\mathrm{OFF}}\right)$ is uniquely controlled by the gate, which could be very attractive for ultra-short devices.

The JL-SOI technology is then potentially interesting for future ultra-scaled devices, due to the simplified technological process without junction engineering and reduced leakage current. JL-SOI MOSFETs have been already fabricated, but experimental studies concerning their sensitivity to radiation are not yet available in literature. In the context of microelectronics characterized by industrial needs for high-reliable circuits in a wide area of applications, it is important to investigate in detail the sensitivity to radiation of the JL-SOI technology with respect to the radiation sensitivity of other more conventional technologies envisaged for high-reliability concerns, such as the inversion-mode (IM) FDSOI (IM-SOI, Fig. 1b) and inversion-mode double-gate (IM-DG) MOSFETs [8-9]. These last devices are very interesting for the nanometer scale, since they present a very good control of parasitic short channel effects (SCE) [10], no parasitic doping fluctuation effects due to the intrinsic nature of the film, increased carrier mobility and drain current [11] and high probability of 
ballistic transport in the channel [12-14].

From a radiation-sensitivity point of view, the high doping level in the film of a JL MOSFET could have a negative impact on its immunity to single events, because floating body effects are expected to be strong. This was confirmed by our previous works [15-16] concerning the radiation-induced transient behavior of JL Double-Gate (JL-DG) MOSFETs. In spite of its double-gate configuration, JL-DGFET should be more sensitive to radiation than IM-DGFET for which the channel is intrinsic. The transient response of IM-DG devices under heavy-ion irradiation has been studied by 3-D numerical simulation in [17-20]. These previous studies demonstrated that IM-DG shows a better resistance to radiation than IM-SOI, due to the enhanced control of the film potential by the two connected gates which reduces floating-body effects. The bipolar amplification of JL-DG was studied in [15] and compared to that of IM-DG with similar geometrical parameters. In that previous work, we have shown that JL-DG is characterized by a higher bipolar gain than IM-DG, due to stronger floatingbody effects. In addition, results obtained in [16] have shown that JL-DG SRAM cell is characterized by a lower critical charge than IM-DG cell, but higher than IM-SOI SRAM.

In the present work, we investigate, for the first time, the radiation sensitivity of JL-SOI, in terms of bipolar gain of individual devices and SEU sensitivity of six-transistor (6T) SRAM cells. A detailed comparison with JL-DG, IM-SOI and IM-DG devices and 6T SRAM cells has been also performed.

\section{Simulation details}

Figure 1 shows the schematic 3-D descriptions of the simulated JL-SOI and IM-SOI devices. JL-SOI devices are designed with $100 \mathrm{~nm}$ gate width, $6 \mathrm{~nm}$-thick silicon film and 0.9 nm-thick gate oxide. The entire silicon film is uniformly n-type doped at $1 \times 10^{19} \mathrm{~cm}^{-3}$; there are no highly-doped source/drain regions. A $10 \mathrm{~nm}$-thick buried oxide (BOX) and a thick silicon substrate doped at $5 \times 10^{18} \mathrm{~cm}^{-3}$ have been also considered. The very thin buried oxide is necessary to minimize the short channel effects in this JL-SOI device. It should be noted that the channel thickness has to be sufficiently small in order to make possible the complete depletion of the silicon film and to be able to cut-off the device [4]. This condition is satisfied for the doping level and the film thickness considered here. IM-SOI devices (Fig. 1b) have an intrinsic channel; source and drain regions are highly n-type doped and the doping profile in these regions is uniform. The silicon film, BOX and silicon substrate of IM-SOI have the 
same geometrical parameters as those of JL-SOI. The silicon substrate is lowly-doped at $10^{16}$ $\mathrm{cm}^{-3}$. IM-DG and JL-DG structures are based on real devices reported in [21]. The silicon film of IM-DG and JL-DG has the same geometrical parameters and doping profiles as the silicon film of IM-SOI and JL-SOI, respectively, with the notable exception that two gates connected together control the channel potential. These four different structures have been first simulated with $20 \mathrm{~nm}$-channel length, considering a power supply voltage of $0.75 \mathrm{~V}$. These devices have been calibrated on the ITRS LP [22]; to facilitate the comparison, the gates work functions have been finely tuned to achieve the same $\mathrm{I}_{\mathrm{OFF}}$ for all devices. Secondly, additional simulations have been carried out for other channel lengths and power supply voltages.

3-D numerical simulations have been performed with the DESSIS device simulator from Synopsis Inc. [23]. The main models used in simulation are: SRH and Auger recombination models, Fermi-Dirac carrier statistics, hydrodynamic model for the carrier transport, mobility model including the dependence on the carrier energy, lattice temperature and doping level and impact ionization model depending on carrier energy. Realistic values have been used for the main electrical parameters of the models used in simulation, such as electrons and holes lifetimes and low field carrier mobility. The ion strike was simulated using the DESSIS HeavyIon module [23]. The electron-hole pair column created in the device by the ion strike is modeled using a carrier-generation function which has a Gaussian radial distribution with a characteristic radius of $20 \mathrm{~nm}$ and a Gaussian time distribution, centered on $10 \mathrm{ps}$ and having a characteristic width of 2 ps.

\section{Static characteristics of individual devices}

The simulated steady-state drain current characteristics of JL-SOI, IM-SOI, JL-DG and IM-DG are plotted in Fig. 2. The devices have the same off-state current, but different subthreshold swings and on-state currents. While double-gate devices (both JL-DG and IMDG) have near ideal subthreshold swings $(65 \mathrm{mV} / \mathrm{dec})$, SOI devices have a much higher subthreshold swing $(90 \mathrm{mV} / \mathrm{dec})$ because the single-gate configuration reduces the control by the gate of the channel potential and increases the parasitic short-channel effects compared to a double-gate configuration. JL-SOI has the lowest on-state current because the highly-doped silicon film degrades the mobility. The highest on-state current is obtained in IM-DG, due to the combination of a double-gate structure and an intrinsic channel; this structure has the advantage to maximize the carrier mobility and the gate-channel coupling. 


\section{Single-event transients}

\subsection{Drain current, collected charge and bipolar gain}

Figure 3 shows the drain current transient resulting from an ion hit in the channel center of JL-SOI and IM-SOI devices. Time variations of the collected charge are also reported on the same figure. The drain current transient peak and width are higher in JL-SOI than in IM-SOI, probably due to a higher bipolar gain. In addition, the drain current decay after the ion strike is slower for JL-SOI than for IM-SOI. The reason is that the floating body effects are more important in JL-SOI than in IM-SOI, due to the high doping level in the JL-SOI film (since the device channel is intrinsic in IM-SOI). The collected charge and the bipolar amplification as function of the ion LET are plotted in Figs. $4 \mathrm{a}$ and $4 \mathrm{~b}$, respectively. The values obtained in [15] for JL-DG and IM-DG devices are also reported in these figures for comparison. As expected, the collected charge and the bipolar gain are higher in JL-SOI than in IM-SOI due to stronger FBE. Compared to the JL-DG structure having the same film doping level, JL-SOI device presents a higher bipolar gain for all LET values. This is due to a less effective film potential control in this structure with a single-gate compared to the double-gate control of the JL-DG device. The bipolar gain decreases when the LET increases because the parasitic bipolar transistor enters in the high-injection regime. At very high LET value, the bipolar gain in JL-SOI decreases rapidly and becomes close to the values obtained in IM-SOI, JL-DG and IM-DG.

\subsection{Dependence on the ion hit location}

We also studied the dependence of the bipolar gain on the ion hit location along the channel ( $x$ axis). Several locations are considered between the source contact $(x=0)$ and the drain contact $(x=60 \mathrm{~nm})$, as illustrated in Fig. 5a. The 3-D profile of the heavy ion charge density in the silicon film is also shown in Fig. 5a for an ion hitting the film at $x=30 \mathrm{~nm}$ (channel center). The current transient have been simulated for each $\mathrm{x}$ location and the collected charge was extracted from this transient. The bipolar gain is then obtained at a given LET for each $\mathrm{x}$ value. Figure $5 \mathrm{~b}$ plots the bipolar gain dependence on the ion hit location for the four studied devices. The bipolar gain is always higher in JL-SOI than in IM-SOI, but has similar dependences on the ion hit location for all devices. 


\section{SEU in 6T SRAM cells: critical charge and threshold LET}

For the four technologies, the 6 transistors of the SRAM cell (Fig. 6) were entirely simulated in the 3-D device domain with the Synopsis/DESSIS module and were connected via the Mixed-Mode module [23]. Only the OFF-state NMOS transistor was struck by an ionizing particle (see Fig. 6), this particular case corresponding to the most effective scenario to disturb the cell and to flip its logical state.

Before simulating the SRAM cell, we have determined for each technology the worst-case condition in terms of $x$ location (along the channel) of the ion hit in the OFF-state NMOS. The worst-case location is the $\mathrm{x}$ location for which the collected charge is the highest. Then, the SEU LET threshold obtained in this point will be the smallest LET for which the SRAM cell flips. To find the worst-case location, the current transient is simulated for each $\mathrm{x}$ location and the collected charge is extracted from this transient. The bipolar gain is then obtained at a given LET for each $\mathrm{x}$ value (according to a similar analysis to that presented in section 4.2). The worst-case $\mathrm{x}$ locations were found to be $\mathrm{x}=35 \mathrm{~nm}$ for JL-SOI and $\mathrm{x}=40 \mathrm{~nm}$ for IM-SOI devices. In the following, we used these worst-case locations for the SRAM cell simulations.

\subsection{Simulation of SRAM cells}

In a first time, we simulated the behavior of the SRAM cell built with JL-SOI transistors when an ionizing particle strikes the OFF-state NMOS transistor. Figure 7a shows the time variation of voltages extracted at nodes " $\mathrm{A}$ " and " $\mathrm{B}$ " $\left(\mathrm{V}_{\mathrm{A}}\right.$ and $\left.\mathrm{V}_{\mathrm{B}}\right)$. The corresponding time variations of the current and the collected charge measured at the drain (node "A") of the impacted transistor for three values of the ion LET are shown in Fig. 7b. When the ion LET increases the current peak increases because the capacitive effect induced by the ion is stronger [16]. The charge collected at node "A" also increases (it proportionally depends on the ion LET). The current transient induced by the ionizing particle disturbs the voltages of nodes " $\mathrm{A}$ " and " $\mathrm{B}$ " and the disturbance is stronger when the ion LET increases. Figure 7a shows that, for LET $=0.5$ and $0.8 \mathrm{MeV} \cdot \mathrm{cm}^{2} / \mathrm{mg}, \mathrm{V}_{\mathrm{A}}$ and $\mathrm{V}_{\mathrm{B}}$ are disturbed, but their values do not change at the end of the transient. For these LET values the state of the SRAM cell is not modified (the cell does not flip). On the contrary, for LET $=1.5 \mathrm{MeV} \cdot \mathrm{cm}^{2} / \mathrm{mg}$, the values of $\mathrm{V}_{\mathrm{A}}$ and $\mathrm{V}_{\mathrm{B}}$ change with respect to their initial value and the SRAM cell flips. In this case, the ion LET is sufficiently high to induce a collected charge larger than the critical charge of the 
cell and to cause the SRAM cell upset.

Secondly, we investigate the behavior of SRAM cells made up of JL-SOI and IM-SOI. Time variations of the voltages $V_{A}$ and $V_{B}$ for $L E T=3 \mathrm{MeV} \cdot \mathrm{cm}^{2} / \mathrm{mg}$ are shown in Fig. 8 for JL-SOI and IM-SOI SRAM cells. For this LET value, the JL-SOI SRAM cell has flipped, while the IM-SOI SRAM cell did not flip. This result gives a first indication on the radiation hardness of JL-SOI technology: the SEU threshold LET of JL-SOI SRAM cell will be lower than that of IM-SOI, which means that the JL-SOI is more sensitive to radiation than the IMSOI technology.

\subsection{Threshold LET and critical charge}

To refine the analysis, we obtained the SEU threshold LET ( $\mathrm{LET}_{\mathrm{th}}$ ) of each cell by varying the ion strike LET until the SRAM cell was observed to upset. As expected, the critical charge $\left(Q_{\text {crit }}=0.126 \mathrm{fC}\right)$ and the threshold LET $\left(\mathrm{LET}_{\mathrm{th}}=1.32 \mathrm{MeV} \cdot \mathrm{cm}^{2} / \mathrm{mg}\right)$ are lower for the JL-SOI SRAM than for IM-SOI SRAM cell $\left(\mathrm{Q}_{\text {crit }}=0.205 \mathrm{fC}\right.$ and $\mathrm{LET}_{\text {th }}=3.15$ $\mathrm{MeV} \cdot \mathrm{cm}^{2} / \mathrm{mg}$, [16]). In order to explain these results, we remind that $\mathrm{Q}_{\text {crit }}$ increases with the equivalent capacitance of the struck node $\left(\mathrm{C}_{\mathrm{N}}\right)$, with the supply voltage $\left(\mathrm{V}_{\mathrm{DD}}\right)$ and with the maximum current of the on-state PMOS transistor ( $\mathrm{I}_{\mathrm{PMOS}}$ ), as explained in [24]. In our study, all cells are operating at the same $\mathrm{V}_{\mathrm{DD}} . \mathrm{C}_{\mathrm{N}}$ is the same for JL-SOI and IM-SOI SRAM cells, but $\mathrm{I}_{\mathrm{PMOS}}$ is lower in JL-SOI than in IM-SOI; this then explains why $\mathrm{Q}_{\text {crit }}$ is lower in JL-SOI than in IM-SOI SRAM cell. $\mathrm{Q}_{\text {crit }}$ and LET $_{\text {th }}$ of JL-SOI are also lower than those corresponding to JL-DG $\left(\mathrm{Q}_{\text {crit }}=0.309 \mathrm{fC}\right.$ and $\left.\mathrm{LET}_{\mathrm{th}}=3.64 \mathrm{MeV} \cdot \mathrm{cm}^{2} / \mathrm{mg}\right)$ and IM-DG $\left(\mathrm{Q}_{\text {crit }}=0.51 \mathrm{fC}\right.$ and $\mathrm{LET}_{\mathrm{th}}=7.48 \mathrm{MeV} \cdot \mathrm{cm}^{2} / \mathrm{mg}$ ) obtained in [16].

\subsection{Dependence on the channel length, power supply and film doping level}

To study more in depth the radiation hardness of these SRAM cells, we investigated the dependence of the SEU threshold LET on the channel length, power supply and film doping level (for JL-SOI SRAM cells). Figure 9 presents the threshold LET values obtained for two channel lengths ( $\mathrm{L}=20 \mathrm{~nm}$ and $\mathrm{L}=50 \mathrm{~nm})$ and for all devices considered in this work. $50 \mathrm{~nm}$ gate length devices have the same geometrical and doping parameters as those of 20-nm gate length devices, excepted for the channel lengths. Our simulations showed that the critical charge and the threshold LET (Fig. 9) are reduced when the channel length decreases for all technologies, in accordance with previous results [25]. 
SRAM simulations have been performed on 20-nm length JL-SOI structures for different power supply values (all geometrical and doping parameters are the same as in section 2, only the power supply varies). As expected from theoretical predictions (explained before), the critical charge increases with the power supply. Our simulations give $\mathrm{Q}_{\text {crit }}=0.175 \mathrm{fC}$ for $\mathrm{V}_{\mathrm{DD}}=0.9 \mathrm{~V}$ and $\mathrm{Q}_{\text {crit }}=0.21 \mathrm{fC}$ for $\mathrm{V}_{\mathrm{DD}}=1.1 \mathrm{~V}$. The threshold LET also increases: we obtained $\mathrm{LET}_{\mathrm{th}}=1.8 \mathrm{MeV} \cdot \mathrm{cm}^{2} / \mathrm{mg}$ for $\mathrm{V}_{\mathrm{DD}}=0.9 \mathrm{~V}$ and $\mathrm{LET}_{\mathrm{th}}=2.04 \mathrm{MeV} \cdot \mathrm{cm}^{2} / \mathrm{mg}$ for $\mathrm{V}_{\mathrm{DD}}=1.1 \mathrm{~V}$.

SRAM simulations have been also conducted on 20-nm gate length JL-SOI technology with different film doping levels. Three additional doping levels have been considered: $5 \times 10^{18}, 1.5 \times 10^{19} \mathrm{~cm}^{-3}$ and $2 \times 10^{19} \mathrm{~cm}^{-3}$. The gate workfunctions of these devices have been tuned in order to obtain the same $\mathrm{I}_{\text {off }}$ current as the 20 -nm gate length JL-SOI structure having the silicon film doped at $1.5 \times 10^{19} \mathrm{~cm}^{-3}$ (other geometrical and doping parameters are the same as those considered in section 2). Critical charges and threshold LETs, presented in Table I, decrease when the doping level increase from $5 \times 10^{18}$ to $2 \times 10^{19} \mathrm{~cm}^{-3}$. To explain these results, the behavior of individual devices has been analyzed. Our simulations showed that when the film doping of JL-SOI increases, the floating body effects are enhanced, the drain current transient is longer and the impact ionization increase. These results are in perfect agreement with those obtained in [15] for JL-DG MOSFETs. Then, both the collected charge and the bipolar amplification increase, which leads to a lower critical charge needed to flip the SRAM cell and to a lower threshold LET.

\section{Conclusion}

In this paper we have presented the transient response to heavy ion irradiation of JL-SOI devices and SRAM cells. Our results show that the bipolar amplification is higher in JL-SOI devices than in conventional IM-SOI because the floating body effects and the impact ionization are enhanced due to the highly doped channel. In addition, JL-SOI SRAM cell is characterized by a lower critical charge and threshold LET than IM-SOI cell. Compared to the JL-DG structure having the same film doping level, JL-SOI device presents a higher bipolar gain, lower critical charge and lower threshold LET. This is due to a less effective film potential control in JL-SOI with a single gate compared to the double-gate control of the JLDG device. In addition, when the film doping of individual devices increases the radiation hardness of the JL-SOI SRAM cell is degraded. From these results, we could expect a worse immunity to single-event phenomena of JL-SOI devices and circuits compared to that of IM- 
SOI and JL-DG MOSFETs.

\section{References}

[1] R.C. Baumann, "Radiation-Induced Soft Errors in Advanced Semiconductor Technologies", IEEE Trans. Device Mater. Reliab., vol. 5, no. 3, pp. 305-316, 2005.

[2] J.L. Autran and D. Munteanu, "Soft Errors: from particles to circuits", Taylor \& Francis/CRC Press, 439 p., 2015.

[3] P. Roche, J.L. Autran, G. Gasiot, D. Munteanu, "Technology downscaling worsening radiation effects in bulk: SOI to the rescue”, IEEE International Electron Device Meeting (IEDM), pp. 766-769, 2013.

[4] C.-W. Lee, A. Afzalian, N.D. Akhavan, R. Yan, I. Ferain, J P. Colinge, "Junctionless multigate fieldeffect transistor,” Applied Physics Letters, vol. 94, p. 053511, 2009.

[5] J.P. Colinge, C.-W. Lee, A. Afzalian, N.D. Akhavan, R. Yan, I. Ferain, P. Razavi, B. O’Neill, A. Blake, M. White, A.-M. Kelleher, B. McCarthy, R. Murphy, "Nanowire transistors without junctions," Nature Nanotechnology, vol. 5, 2010.

[6] A. Kranti, R. Yan, C.-W. Lee, I. Ferain, R. Yu, N.D. Akhavan, P. Razavi, J.P. Colinge, "Junctionless Nanowire Transistor (JNT): Properties and Design Guidelines”, in Proc. European Solid State Device Research Conf. (ESSDERC), pp. 357-360, 2010.

[7] J.P. Colinge, C.-W. Lee, I. Ferain, N.D. Akhavan, R. Yan, P. Razavi, R. Yu, A. Nazarov, R.T. Doria, "Reduced electric field in junctionless transistors," Applied Physics Letters, vol. 96, p. 073510, 2009.

[8] S. Harrison, D. Munteanu, J.L. Autran, A. Cros, R. Cerutti, T. Skotnicki, "Electrical characterization and modelling of high-performance SON DG MOSFETs”, in Proc. European Solid-State Device Research Conference (ESSDERC), pp. 373-376, 2004.

[9] D. Munteanu, J.L. Autran, S. Harrison, "Quantum short-channel compact model for the threshold voltage in Double-Gate MOSFETs with high-k permittivity gate dielectrics", J. Non-Crystalline Solids, vol. 351, Issue 21-23, p. 1911-1918, 2005.

[10] D. Munteanu, J.L. Autran, S. Harrison, K. Nehari, O. Tintori, T. Skotnicki, "Compact Model of the Quantum Short-Channel Threshold Voltage in Symmetric Double-Gate MOSFET", Molecular Simulation, vol. 31, no. 12, pp. 831-837, 2005.

[11] D. Hisamoto, "FD/DG-SOI MOSFET-A viable approach to overcoming the device scaling limit", in Int. Electron Devices Meeting Tech. Dig., pp. 429-432, 2001.

[12] V. Barral, T. Poiroux, M. Vinet, J. Widiez, B. Previtali, P. Grosgeorges, G. Le Carval, S. Barraud, J.L. Autran, D. Munteanu, S. Deleonibus, "Experimental determination of the channel backscattering coefficient on 10-70 nm-metal-gate Double-Gate transistors", Solid State Electronics, vol. 51, no. 4, pp. 537-542, 2007.

[13] D. Munteanu and J.L. Autran, "Two-dimensional Modeling of Quantum Ballistic Transport in Ultimate Double-Gate SOI Devices", Solid State Electronics, vol. 47, pp. 1219-1225, 2003.

[14] J.L. Autran and D. Munteanu, "Simulation of electron transport in nanoscale independent-gate DG 
devices using a full 2D Green's function approach”, J. Comput. Theoret. Nanosci., vol. 5, pp. 1120-1127, 2008.

[15] D. Munteanu and J.L. Autran, "3-D Numerical Simulation of Bipolar Amplification in Junctionless Double-Gate MOSFETs Under Heavy-Ion Irradiation”, IEEE Trans. Nucl. Sci., vol. 59, no. 4, pp. 773780, 2012.

[16] D. Munteanu and J.L. Autran, "Radiation sensitivity of junctionless double-gate 6T SRAM cells investigated by 3-D numerical simulation”, Microelectronics Reliability, vol. 54, pp. 2284-2288, 2014.

[17] D. Munteanu, V. Ferlet-Cavrois, J.L. Autran, P. Paillet, J. Baggio, O. Faynot, C. Jahan, L. Tosti, "Investigation of Quantum Effects in Ultra-Thin Body Single- and Double-Gate Devices Submitted to Heavy Ion Irradiation", IEEE Trans. Nucl. Sci., vol. 53, no. 6, pp. 3363-3371, 2006.

[18] D. Munteanu, J.L. Autran, V. Ferlet-Cavrois, P. Paillet, J. Baggio, K. Castellani, "3-D Quantum Numerical Simulation of Single-Event Transients in Multiple-Gate Nanowire MOSFETs", IEEE Trans. Nucl. Sci., vol. 54, no. 4, pp. 994-1001, 2007.

[19] D. Munteanu and J.L. Autran, "Modeling and Simulation of Single-Event Effects in Digital Devices and ICs”, IEEE Trans. Nucl. Sci., vol. 55, no. 4, pp. 1854-1878, 2008.

[20] D. Munteanu and J.L. Autran, "3-D Simulation Analysis of Bipolar Amplification in Planar Double-Gate and FinFET with Independent Gates”, IEEE Trans. Nucl. Sci., vol. 56, no. 4, pp. 2083-2090, 2009.

[21] M. Vinet, T. Poiroux, J. Widiez, J. Lolivier, B. Previtali, C. Vizioz, B. Guillaumot, Y. Le Tiec, P. Besson, B. Biasse, F. Allain, M. Cassé, D. Lafond, J.-M. Hartmann, Y. Morand, J. Chiaroni, S. Deleonibus, "Bonded Planar Double-Metal-Gate NMOS Transistors Down to $10 \mathrm{~nm}$ ”, IEEE Electron Dev. Lett., vol. 26, no. 5, pp. 317-319, 2005.

[22] International Technology Roadmap for Semiconductors (ITRS), available online: http://public.itrs.net/.

[23] Synopsys Sentaurus TCAD tools, Available online: http://www.synopsys.com/products/tcad/tcad.html.

[24] S.M. Jahinuzzaman, M. Sharifkhani, M. Sachdev, "An Analytical Model for Soft Error Critical Charge of Nanometric SRAMs", IEEE Trans. Very Large Scale Integr. (VLSI) Syst., vol. 17, pp. 1187-1195, 2009.

[25] P. Shivakumar, M. Kistler, S.W. Keckler, D. Burger, L. Alvisi, "Modeling the Effect of Technology Trends on the Soft Error Rate of Combinational Logic", in Proceedings of the International Conference on Dependable Systems and Networks, pp. 389-398, 2002. 


\section{Figure and Table Captions}

Fig. 1. Schematic description of the simulated JL-SOI (a) and IM-SOI (b) MOSFETs. For a better view spacers and isolation oxides are not shown. The doping level distribution in each device is shown and the main geometrical parameters are defined. The position of the ion strike is indicated by the arrow; the ion strikes vertically in the middle of the channel and in a direction parallel to the $\mathrm{z}$ axis.

Fig. 2. Drain current as a function of gate voltage for JL-SOI, IM-SOI, JL-DG and IM-DG. The gate workfunction of each device has been tuned to obtain the same $\mathrm{I}_{\mathrm{OFF}}$.

Fig. 3. Drain current transient and collected charge in JL-SOI and IM-SOI for an ion hit in the channel center.

Fig. 4. Collected charge and bipolar amplification as function of LET in JL-SOI and IM-SOI MOSFETs for an ion hit in the channel center.

Fig. 5. (a) 3-D profile of heavy-ion charge density in the silicon film of JL-SOI for an ion hit at $\mathrm{x}=30 \mathrm{~nm}$ and $\mathrm{LET}=1 \mathrm{MeV} \cdot \mathrm{cm}^{2} / \mathrm{mg}$. Other positions for the ion strike considered in this work are also indicated. For a better view of the film, gate material, spacers and isolation oxide are not shown. (b) Bipolar gain in JL-SOI, IM-SOI, JL-DG and IM-DG as a function of the ion hit location.

Fig. 6. Schematics of the SRAM cell simulated in this work. The particle hits the OFF-state 
NMOS transistor (NMOS 2).

Fig. 7. JL-SOI SRAM cells: (a) time variation of voltages $V_{A}$ and $V_{B}$ and (b) current transient and collected charge at node " $\mathrm{A}$ ".

Fig. 8. Time variation of $V_{A}$ and $V_{B}$ in JL-SOI and IM-SOI SRAM cells at LET=3 $\mathrm{MeV} \cdot \mathrm{cm}^{2} / \mathrm{mg}$.

Fig. 9. Threshold LET of JL-SOI, IM-SOI, JL-DG and IM-DG SRAM cells for two channel lengths, $\mathrm{L}=20$ and $50 \mathrm{~nm}$.

Table I. Critical charge and threshold LET in 20-nm gate length JL-SOI SRAM cells for different film doping levels of individual devices. 


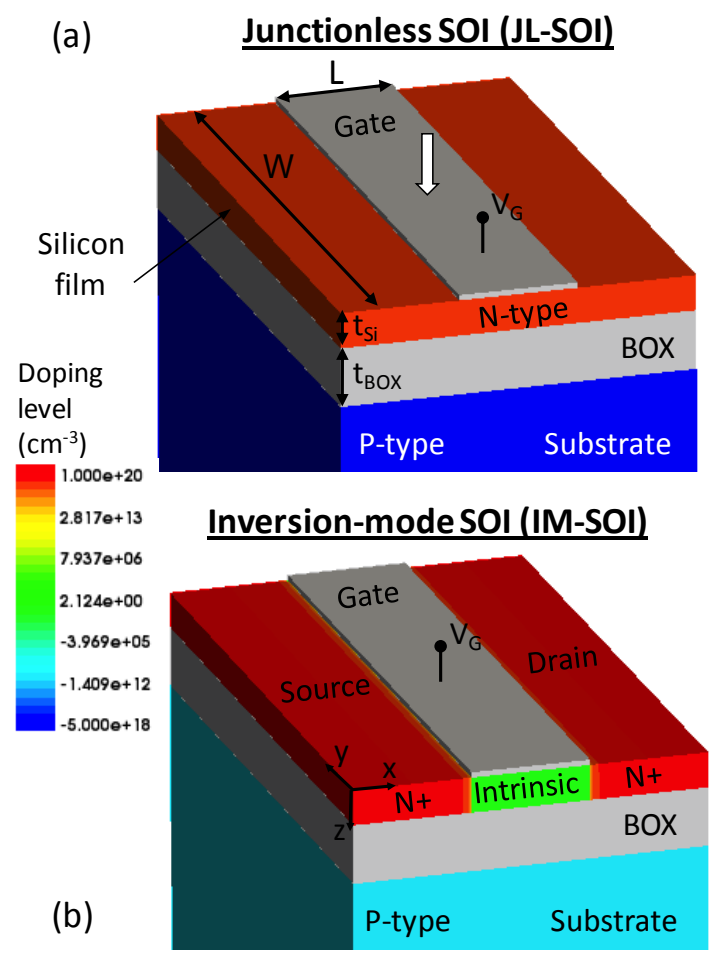

Figure 1. Munteanu and Autran. 


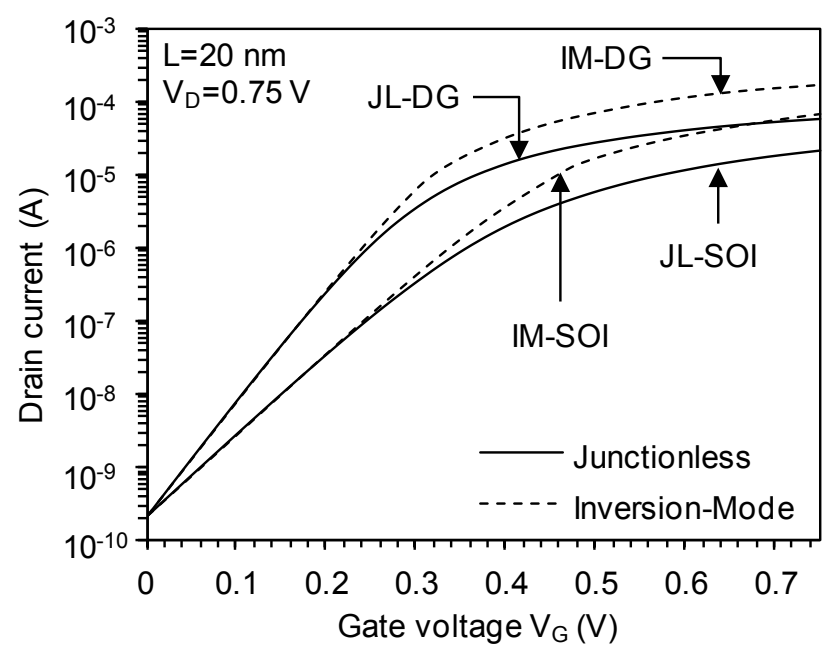

Figure 2. Munteanu and Autran. 


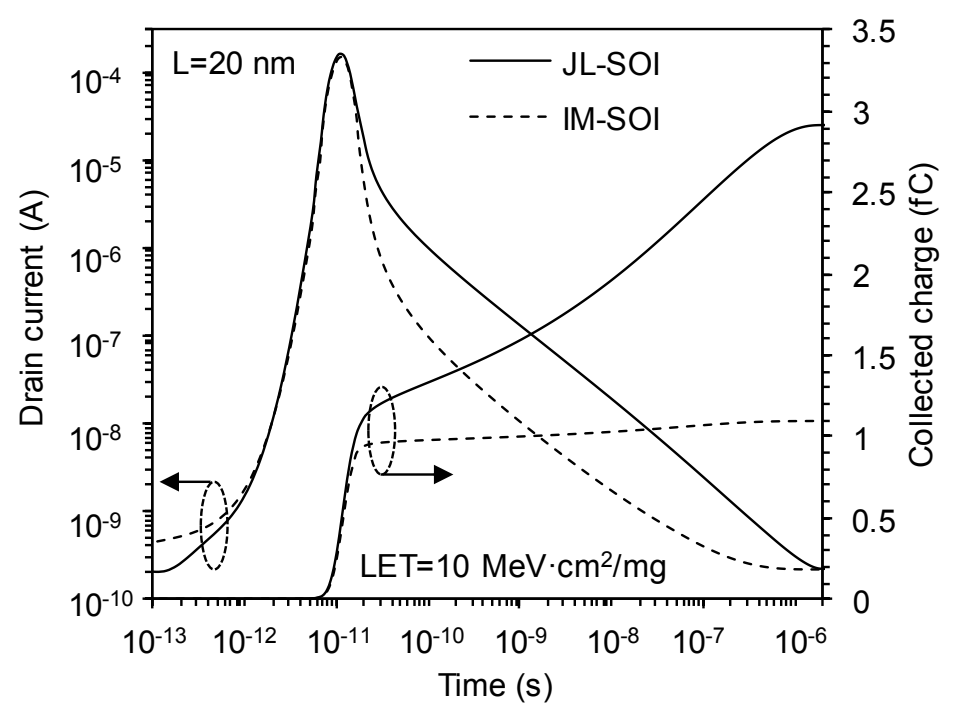

Figure 3. Munteanu and Autran. 


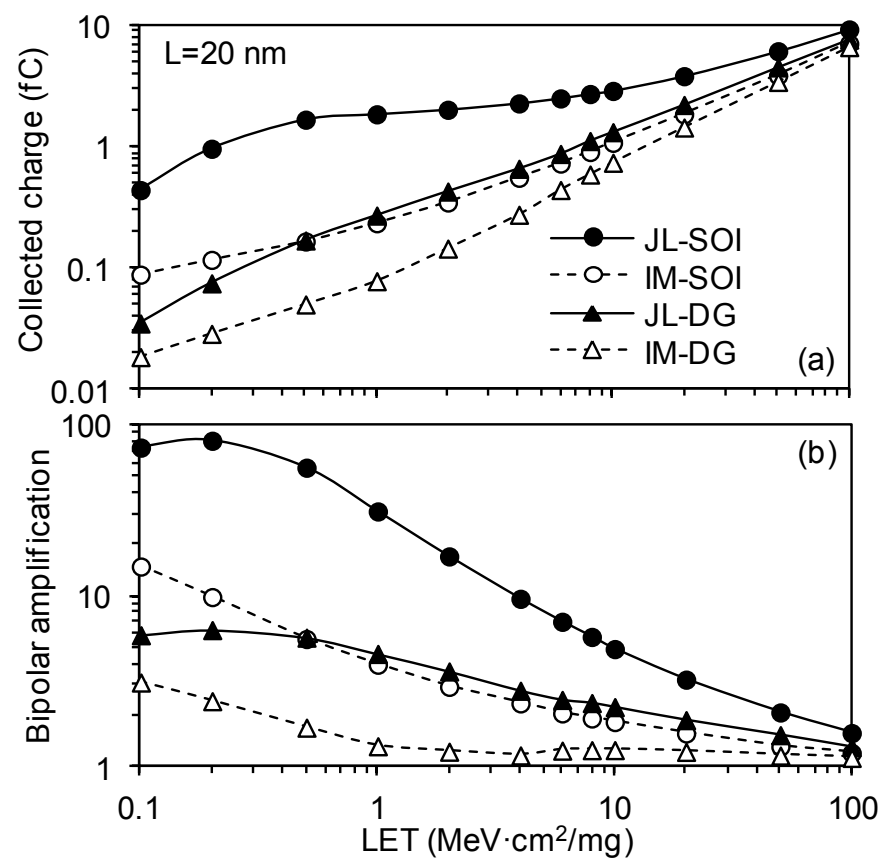

Figure 4. Munteanu and Autran. 
Heavy-lon Charge Density $\left(\mathrm{cm}^{-3}\right)$

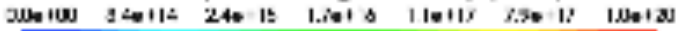
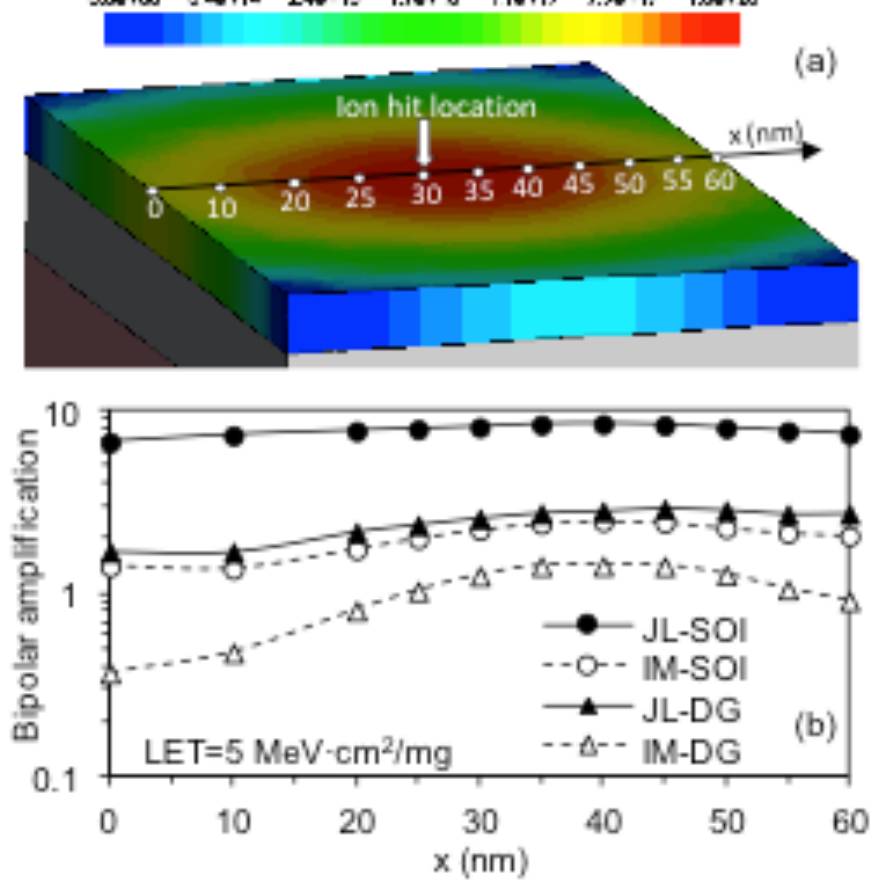

Figure 5. Munteanu and Autran. 


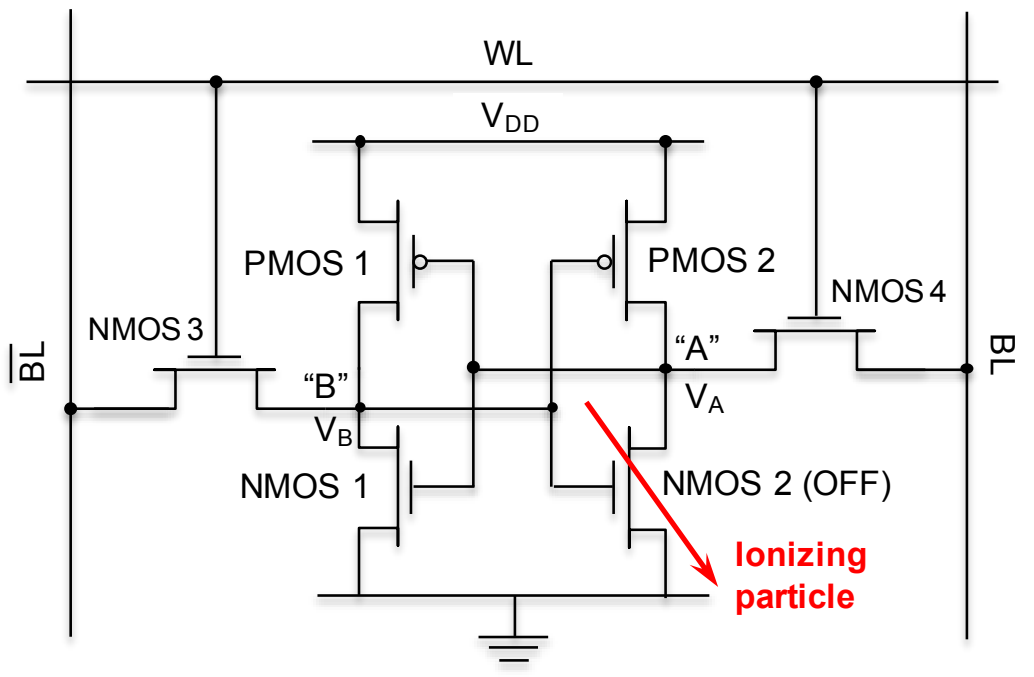

Figure 6. Munteanu and Autran. 


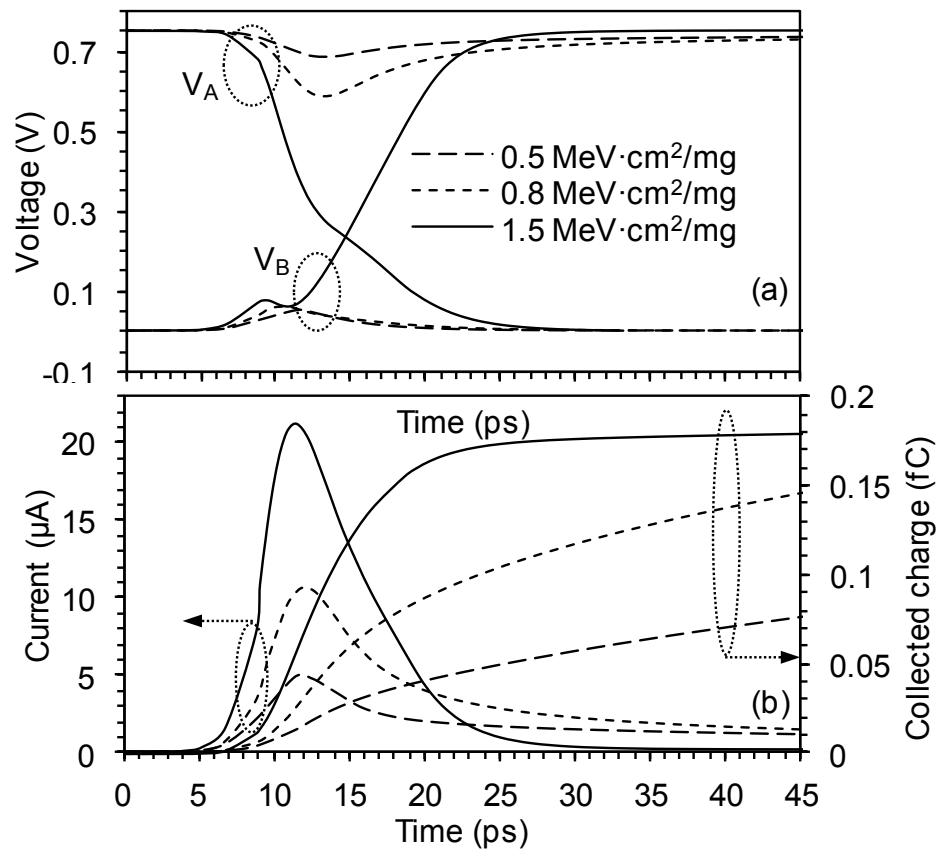

Figure 7. Munteanu and Autran. 


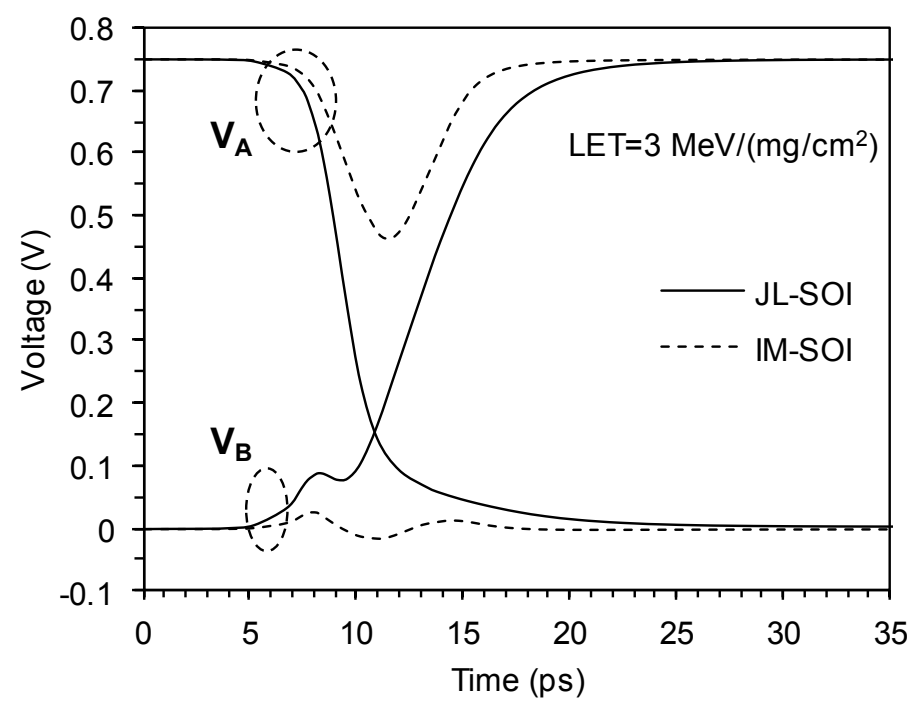

Figure 8. Munteanu and Autran. 


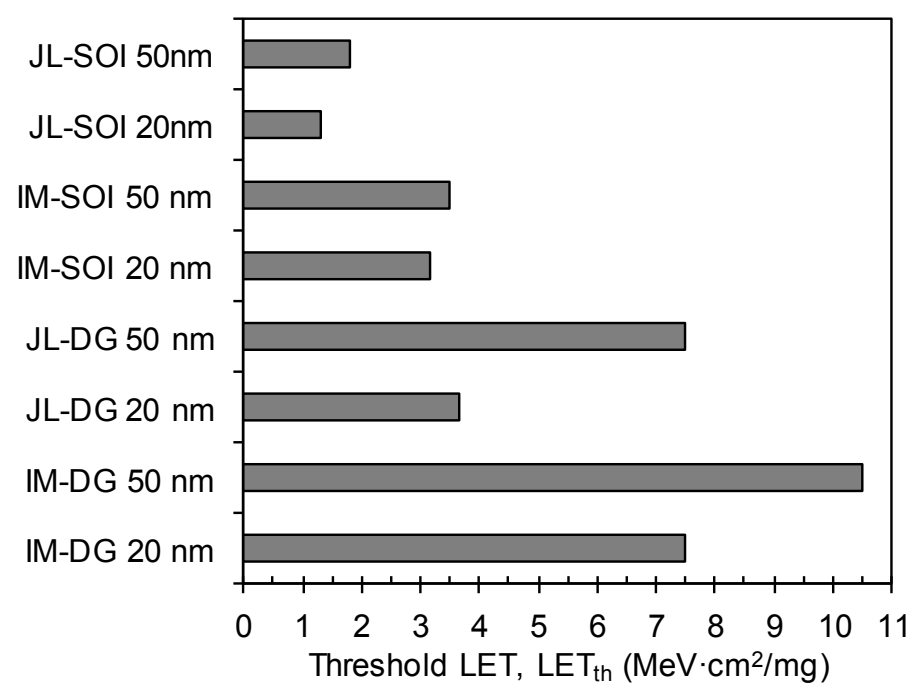

Figure 9. Munteanu and Autran. 
Table I.

\begin{tabular}{|c|c|c|}
\hline $\begin{array}{c}\text { Film doping level } \\
\left(\mathrm{cm}^{-3}\right)\end{array}$ & $\begin{array}{c}\text { Critical charge } \\
(\mathrm{fC})\end{array}$ & $\begin{array}{c}\text { Threshold LET } \\
\left(\mathrm{MeV} \cdot \mathrm{cm}^{2} / \mathrm{mg}\right)\end{array}$ \\
\hline $5 \times 10^{18}$ & 0.158 & 1.95 \\
\hline $1 \times 10^{19}$ & 0.126 & 1.32 \\
\hline $1.5 \times 10^{19}$ & 0.110 & 0.86 \\
\hline $2 \times 10^{19}$ & 0.104 & 0.75 \\
\hline
\end{tabular}

